\title{
COMPLEX FOURIER ANALYSIS ON A NILPOTENT LIE GROUP
}

\author{
BY \\ ROE GOODMAN( $\left.{ }^{1}\right)$
}

\begin{abstract}
Let $G$ be a simply-connected nilpotent Lie group, with complexification $G_{c}$. The functions on $G$ which are analytic vectors for the left regular representation of $G$ on $L_{2}(G)$ are determined in this paper, via a dual characterization in terms of their analytic continuation to $G_{c}$, and by properties of their $L_{2}$ Fourier transforms. The analytic continuation of these functions is shown to be given by the Fourier inversion formula. An explicit construction is given for a dense space of entire vectors for the left regular representation. In the case $\boldsymbol{G}=\boldsymbol{R}$ this furnishes a group-theoretic setting for results of Paley and Wiener concerning functions holomorphic in a strip.
\end{abstract}

Introduction. Let $G$ be a simply-connected nilpotent Lie group. Since $G$ is a separable, type I, unimodular group [1], there exists by the general Plancherel theorem [3] a unique Borel measure $\mu$ on the space $\hat{G}$ of equivalence classes of irreducible unitary representations of $G$ with the following properties:

(i) (Harmonic analysis). Fix a $\mu$ measurable cross-section $\xi \rightarrow \pi^{\xi}$ from $\hat{G}$ to concrete irreducible unitary representations $\left(\pi^{\xi} \in \xi\right)$. Then a function $f \in L_{2}(G)$ has a Fourier transform $\hat{f}$ defined $\mu$-a.e. on $\hat{G}$, such that $\hat{f}(\xi)$ is a Hilbert-Schmidt operator on the space $\mathscr{H}\left(\pi^{\xi}\right)$ of $\pi^{\xi}$. The map $\xi \rightarrow \hat{f}(\xi)$ is a $\mu$-measurable field of operators, and if $f \in L_{1} \cap L_{2}$ then, for a.e. $\xi$,

$$
\hat{f}(\xi)=\int_{G} f(g) \pi^{\xi}(g) d g .
$$

(ii) (Harmonic synthesis). If $f_{1}, f_{2} \in L_{2}(G)$, then

$$
\left(f_{1} \star f_{2}^{*}\right)(e)=\int_{\hat{G}} \operatorname{tr}\left(\hat{f}_{1}(\xi) \hat{f}_{2}(\xi)^{*}\right) d \mu(\xi),
$$

where $f^{*}(g)=\overline{f\left(g^{-1}\right)}$ (the bar denoting complex conjugation), and tr denotes the canonical semifinite trace on the ring of all bounded operators on $\mathscr{H}\left(\pi^{\xi}\right)$.

If $f$ has sufficiently many derivatives in $L_{2}(G)$, then $f$ can be written as $f_{1} \star f_{2}^{*}$, with $f_{j} \in L_{2}(G)$ [15]. Hence by $(0.2), \hat{f}(\xi)$ will be a trace-class operator for almost all

Received by the editors October 13, 1970.

AMS 1970 subject classifications. Primary 22E30, 22E45; Secondary 30A78, 22E25.

Key words and phrases. Nilpotent Lie group, Plancherel theorem, Fourier inversion formula, Paley-Wiener theorem, analytic vector, entire vector, analytic continuation of representations.

( $\left.{ }^{1}\right)$ This research was supported in part by NSF grant GP 22822.

Copyright (C) 1971, American Mathematical Society 
$\xi$, and $f$ may be expressed in terms of $\hat{f}$ by the Fourier inversion formula:

$$
f(g)=\int_{\hat{G}} \operatorname{tr}\left(\pi^{\xi}\left(g^{-1}\right) \hat{f}(\xi)\right) d \mu(\xi) .
$$

In this paper we want to use the general Plancherel theorem to correlate analyticity properties of functions on $G$ with suitable properties of their Fourier transforms. This was first done for the additive group of reals $\boldsymbol{R}$ by Paley and Wiener [12]. In this case $\hat{G}$ may be parametrized by $\boldsymbol{R}$, with $\pi^{\xi}, \xi \in \boldsymbol{R}$, the onedimensional representation $x \rightarrow \exp (i \xi x)$. For each fixed $\xi$ the function

$$
x \rightarrow \operatorname{tr}\left(\pi^{\xi}(-x) \hat{f}(\xi)\right)=e^{-i \xi x} \hat{f}(\xi)
$$

extends holomorphically to the complexification $\boldsymbol{C}$ of $\boldsymbol{R}$. Hence $f$ will have an analytic continuation, given by $(0.3)$ with $g$ complex, provided $e^{i z \xi} \hat{f}(\xi)$ remains square-integrable with respect to the Plancherel measure, uniformly for $z$ on compact sets of $\boldsymbol{C}$.

In the noncommutative case a new phenomenon occurs. Namely, the function $g \rightarrow \operatorname{tr}\left(\pi^{\xi}\left(g^{-1}\right) T\right)$ is not analytic for an arbitrary trace-class operator $T$ on $\mathscr{H}\left(\pi^{\xi}\right)$, since the matrix elements $g \rightarrow\left(\pi^{\xi}(g) u, v\right), u, v \in \mathscr{H}\left(\pi^{\xi}\right)$, are only continuous in general. To treat analyticity properties of $L_{2}$ functions on $G$ via the Fourier transform, we need the following notions:

Let $\mathfrak{g}$ be the Lie algebra of $G, \mathfrak{g}_{c}$ the complexification of $\mathfrak{g}$, and $G_{c}$ a simplyconnected group with Lie algebra $\mathfrak{g}_{c}$. Since $\mathfrak{g}_{c}$ is nilpotent, we may assume that $G$ is the analytic subgroup exp $\mathfrak{g}$ of $G_{c}\left(\exp : \mathfrak{g}_{c} \rightarrow G_{c}\right)$. Let $\left\{X_{j}\right\}, 1 \leqq j \leqq d$, be an ordered Jordan-Hölder basis for $\mathrm{g}$, i.e. if

$$
\mathfrak{h}_{k}=\operatorname{span}\left\{X_{j}\right\}_{j} \leqq k
$$

then

$$
\left[\mathfrak{g}, \mathfrak{h}_{k}\right] \subset \mathfrak{h}_{k-1}, \quad k=1,2, \ldots, d .
$$

If $\pi$ is a unitary representation of $G$ on a Hilbert space $\mathscr{H}(\pi)$, let $\mathscr{H}^{\omega}(\pi)$ denote the space of analytic vectors for $\pi$, and let the subspaces $\mathscr{H}_{t}^{\omega}(\pi) \subset \mathscr{H}^{\omega}(\pi), t>0$, be defined as in [5] relative to the basis $\left\{X_{j}\right\}$ (cf. $\S 1$ ). Then $\mathscr{H}^{\omega}(\pi)=\bigcup_{t>0} \mathscr{H}_{t}^{\omega}(\pi)$, and we call $\mathscr{H}_{\infty}^{\omega}(\pi)=\bigcap_{t>0} \mathscr{H}_{t}^{\omega}(\pi)$ the space of entire vectors for $\pi .\left(\mathscr{H}_{t}^{\omega}(\pi)\right.$ is dense in $\mathscr{H}^{\omega}(\pi)$ for some $t>0$ [10], and when $\pi$ is irreducible, we showed that $\mathscr{H}_{\infty}^{\omega}(\pi)$ is dense in $\mathscr{H}(\pi)[6]$.

On the space $\mathscr{H}^{\omega}(\pi)$ the representation $\pi$ can be analytically continued to a local representation $\pi_{\omega}$ of $G_{c}$ [5, Proposition 2.3]; if $g=\exp X$, then $\pi_{\omega}(g)$ is defined on the space $\bigcup_{t>|X|} \mathscr{H}_{t}^{\omega}(\pi)$. Here we define the norm $|X|$ relative to the basis $\left\{X_{j}\right\}$ by $\left|\sum a_{j} X_{j}\right|=\sum\left|a_{j}\right|, a_{j} \in C$. Thus if $T$ is a bounded operator on $\mathscr{H}(\pi)$ such that Range $(T) \subseteq \mathscr{H}_{t}^{\omega}(\pi)$, then $\gamma \rightarrow \pi_{\omega}(\gamma) T$ is a holomorphic operator-valued function defined for $\gamma \in \Omega_{t}$, where $\Omega_{t}=\left\{\exp X ; X \in \mathfrak{g}_{c},|X|<t\right\}$.

Let now $L$ be the left regular representation of $G$ on $L_{2}(G)$. We show in this paper that the space $\mathscr{H}^{\omega}(L)$ has the following dual characterizations: 
THEOREM A. A function $f \in L_{2}(G)$ is an analytic vector for the left regular representation $L$ if and only if $f$ extends holomorphically to the region $\Omega_{t} G \subset G_{c}$ and satisfies

$$
\sup _{\gamma \in \Omega_{t}} \int_{G}\left|f\left(\gamma^{-1} g\right)\right|^{2} d g<\infty
$$

for some $t>0, d g$ denoting Haar measure on $G$.

Furthermore, $f$ is an entire vector for $L$ if and only if $f$ is an entire function on $G_{c}$ and $(0.4)$ holds for all $t>0$. The analytic continuation $L_{\omega}$ of $L$ acts by left translations.

THEOREM B. A function $f \in L_{2}(G)$ is an analytic vector for the left regular representation if and only if the Fourier transform $\hat{f}$ of $f$ satisfies

$$
\begin{gathered}
\text { Range }(\hat{f}(\xi)) \subseteq \mathscr{H}_{t}^{\omega}\left(\pi^{\xi}\right) \quad \text { a.e. }(\mu), \\
\int_{\hat{G}} \sup _{\gamma \in \Omega_{t}}\left\|\pi_{\omega}^{\xi}(\gamma) \hat{f}(\xi)\right\|_{2}^{2} d \mu(\xi)<\infty
\end{gathered}
$$

for some $t>0$, where $\mu$ is the Plancherel measure on $\hat{G}$ and $\|T\|_{2}$ denotes the HilbertSchmidt norm.

Furthermore, $f$ is an entire vector for $L$ if and only if $(0.5)$ and $(0.6)$ hold for all $t>0$. The Fourier transform of $L_{\omega}(\gamma) f$ is $\pi_{\omega}^{\xi}(\gamma) \hat{f}(\xi)$ a.e. $(\mu)$.

The analytic continuation of $f$ in Theorem $\mathrm{A}$ is furnished by the Fourier inversion formula:

THEOREM C. Let $f \in L_{2}(G)$ be an analytic vector for the left regular representation, $\hat{f}$ its Fourier transform. Then

$$
\int_{\hat{G}} \sup _{\gamma \in \Omega_{t}}\left\|\pi_{\omega}^{\xi}(\gamma) \hat{f}(\xi)\right\|_{1} d \mu(\xi)<\infty
$$

for some $t>0$, where $\|T\|_{1}$ denotes the trace norm. The analytic continuation of $f$ to the region $\Omega_{t} G \subseteq G_{c}$ is given by the absolutely convergent integral

$$
f(\gamma g)=\int_{\hat{G}} \operatorname{tr}\left(\pi_{\omega}^{\xi}\left(\gamma^{-1}\right) \hat{f}(\xi) \pi^{\xi}\left(g^{-1}\right)\right) d \mu(\xi), \quad \gamma \in \Omega_{t}, g \in G .
$$

The main technical tool used to prove Theorems A and B is a "global" form of the semidirect product theorem for spaces of analytic vectors (cf. Corollary 3.1 of [5]), which we state and prove in $\S 1$. This reduces questions about analytic vectors for $G$ to consideration of analytic vectors for the one-parameter subgroups $\left\{\exp t X_{j}\right\}$. In $\S \S 2,3$, and 4 we state and prove somewhat stronger versions of Theorems A, B, and C, respectively.

The "local" versions of these theorems, i.e. with the omission of the characterization of the space of entire vectors, hold for a general type I unimodular Lie group (assuming for simplicity that $G \subseteq G_{c}$, where $G_{c}$ is the simply-connected group with Lie algebra $g_{c}$ ). This can be proved by the same techniques used in the present 
paper. We omit the details, and instead, to establish the special nature of the nilpotent case, we show in $\$ 5$ that the space of entire vectors for the left regular representation is dense in $L_{2}(G)$, when $G$ is a simply-connected nilpotent group. By the results of [5] this fails to hold for large classes of noncompact, nonnilpotent Lie groups.

1. Basic technical theorem. Let $\left\{X_{j}\right\}, 1 \leqq j \leqq d$, be an ordered Jordan-Hölder basis for $\mathfrak{g}$, which will remain fixed throughout the paper. Let $\pi$ be a unitary representation of the group $G$. On the space $\mathscr{H}^{\infty}(\pi)$ of infinitely differentiable vectors for $\pi$ we define the seminorms $\rho_{n}$ (relative to the basis $\left\{X_{j}\right\}$ ) by

$$
\rho_{n}(v)=\max _{1 \leqq j_{k} \leqq d}\left\|\partial \pi\left(X_{j_{1}} \cdots X_{j_{n}}\right) v\right\|,
$$

$\left(\rho_{0}(v)=\|v\|\right)$, where $\partial \pi$ is the representation of the complex enveloping algebra $\mathfrak{u}(\mathfrak{g})_{c}$ on $\mathscr{H}^{\infty}(\pi)$ obtained from $\pi$. The space $\mathscr{H}_{t}^{\omega}(\pi), t>0$, consists of all $C^{\infty}$ vectors $v$ for which

$$
E_{s}(v)=\sum_{n=0}^{\infty} \frac{s^{n}}{n !} \rho_{n}(v)<\infty
$$

when $0<s<t$. Equivalently, by Abel's lemma,

$$
M_{s}(v)=\sup _{n \geqq 0}\left[s^{n} \rho_{n}(v) / n !\right]<\infty
$$

for $0<s<t$. The families of norms $\left\{E_{s}\right\}_{0<s<t}$ and $\left\{M_{s}\right\}_{0<s<t}$ on $\mathscr{H}_{t}^{\omega}(\pi)$ are obviously equivalent.

Let $\pi_{j}$ denote the restriction of $\pi$ to the one-parameter subgroup $G_{j}=\left\{\exp t X_{j}\right\}_{t \in R}$. Let $\mathscr{H}_{t}^{\omega}\left(\pi_{j}\right)$ be defined with respect to the basis $X_{j}$ for the Lie algebra of $G_{j}$, i.e. $v \in \mathscr{H}_{t}^{\omega}\left(\pi_{j}\right)$ if and only if for all $s, 0<s<t$, there exists a constant $C_{s}(v)$ such that $\left\|\partial \pi\left(X_{j}\right)^{n} v\right\| \leqq C_{s}(v) s^{-n} n$ !. (By the Cauchy inequalities, this is equivalent to assuming that the $\mathscr{H}(\pi)$-valued function $\xi \rightarrow \pi\left(\exp \xi X_{j}\right) v, \xi$ real, can be analytically continued to the complex disc $|\zeta|<t$.)

Since $\left\|\partial \pi\left(X_{j}\right)^{n} v\right\| \leqq \rho_{n}(v)$, we have

$$
\mathscr{H}_{t}^{\omega}(\pi) \subseteq \mathscr{H}_{t}^{\omega}\left(\pi_{j}\right), \quad 1 \leqq j \leqq d .
$$

Our basic technical tool in studying the spaces $\mathscr{H}_{t}^{\omega}(\pi)$ is the following converse to (1.2):

THEOREM 1.1. There exist polynomials $p_{j}(t)$, depending only on the structure of $\mathfrak{g}$, such that for any unitary representation $\pi$ of $G$ and all $t>0$, one has

$$
\bigcap_{j=1}^{d} \mathscr{H}_{p_{j}(t)}^{\omega}\left(\pi_{j}\right) \subseteq \mathscr{H}_{t}^{\omega}(\pi)
$$

If $\mathfrak{h}_{j}=\operatorname{span}\left\{X_{k}\right\}_{1 \leqq k \leqq j}$, and if the integers $N_{j}$ are chosen so that $\left(\operatorname{ad} X_{j+1}\right)^{N_{j}+1}\left(\mathfrak{h}_{j}\right)$ $=0$, then the polynomials $p_{j}(t)$ may be taken as

$$
p_{d}(t)=t, \quad p_{j}(t)=C t(1+t)^{m_{j}}, \quad 1 \leqq j<d,
$$

where $m_{j}+1=\prod_{k=j}^{d-1}\left(2 N_{k}+1\right)$ and $C \geqq 1$ is a constant independent of $\pi$. 
Remarks. 1. In case $\mathfrak{g}$ is abelian, then $N_{j}=0, m_{j}=0$. Thus the theorem is best possible in this case, up to the choice of the constant $C$.

2. The proof of Theorem 1.1 shows that the inclusion (1.3) is continuous in the $\mathscr{H}_{t}^{\omega}$ topologies. This could also be inferred from the closed graph theorem, since both sides of (1.3) are Fréchet spaces [5, Proposition 2.1].

Proof of Theorem 1.1. By Theorem 1.1 of [5], we know that $\bigcap_{j=1}^{d} \mathscr{H}^{\infty}\left(\pi_{j}\right)$ $=\mathscr{H}^{\infty}(\pi)$. To obtain the inclusion (1.3) we shall proceed by induction on $\operatorname{dim} \mathrm{g}$, the theorem being trivial when $\operatorname{dim} g=1$, and we shall use the notation and results of $\S 4$ of [6]. Specifically, assume that the theorem is true for nilpotent Lie algebras of dimension $\leqq d$. Let $\operatorname{dim} \mathfrak{g}=d+1$, and take a Jordan-Hölder basis $\left\{X_{j}\right\}_{1 \leqq j \leqq d+1}$ for $\mathfrak{g}$. For convenience in notation, set $Y_{j}=X_{j}, 1 \leqq j \leqq d, X=X_{d+1}$ and $\mathfrak{h}=\operatorname{span}\left\{Y_{1}, \ldots, Y_{d}\right\}$. Let

$$
\tau_{n}(v)=\max _{1 \leqq j_{k} \leqq d}\left\|\partial \pi\left(Y_{j_{1}} \cdots Y_{j_{n}}\right) v\right\|,
$$

for $v \in \mathscr{H}^{\infty}(\pi)$. Then by Lemma 4.2 of [6], we have the a priori estimate

$$
\rho_{n}(v) \leqq \max _{k+m=n}\left\{\sum_{j=0}^{k}\left(\begin{array}{l}
k \\
j
\end{array}\right) a_{m, j} \tau_{m}\left(\partial \pi(X)^{k-j} v\right)\right\}
$$

holding on $\mathscr{H}^{\infty}(\pi)$. Here $a_{m, j}$ are positive numbers depending only on the Lie algebra structure of $\mathfrak{g}$, such that for all $r>0$ and integers $m$, an inequality

$$
\sum_{j=0}^{\infty} \frac{r^{j}}{j !} a_{m, j} \leqq C^{m}\left(1+r^{N}\right)^{m}
$$

holds. (The exponent $N$ in $(1.6)$ is determined by the condition $(\operatorname{ad} X)^{N+1}=0$.)

In order to obtain an a priori estimate of the right side of (1.5) in terms of $\tau_{m}(v)$ and $\left\|\partial \pi(X)^{k} v\right\|$, we observe that by the skew-symmetry of the operators $\partial \pi\left(X_{j}\right)$ one has

$$
\left\|\partial \pi\left(Y_{j_{1}} \cdots Y_{j_{m}} X^{k}\right) v\right\|^{2}=(-1)^{m+k}\left(\partial \pi\left(X^{k} Y_{j_{m}} \cdots Y_{j_{1}} Y_{j_{1}} \cdots Y_{j_{m}} X^{k}\right) v, v\right)
$$

for any vector $v \in \mathscr{H}^{\infty}(\pi)$. Let $D$ be the derivation of $\mathfrak{u}(\mathfrak{g})_{c}$ which coincides with ad $X$ on $g$. For a multi-index $\alpha=\left(j_{1}, \ldots, j_{n}\right)$, with $1 \leqq j_{k} \leqq d$, set $Y_{\alpha}=Y_{j_{1}} \cdots Y_{j_{n}}$. Then by Lemma 4.1 of [6], we may express

$$
D^{k} Y_{\alpha}=\sum_{|\beta|=|\alpha|} C_{\alpha, \beta}^{k} Y_{\beta}
$$

with the coefficients $C_{\alpha, \beta}^{k}$ satisfying $\max _{|\alpha|=n} \sum_{|\beta|=n}\left|C_{\alpha, \beta}^{k}\right| \leqq a_{n, k}, a_{n, k}$ as above. Now by the standard commutation formula

$$
X^{k} Y_{\alpha}=\sum_{j=0}^{k}\left(\begin{array}{l}
k \\
j
\end{array}\right) D^{j}\left(Y_{\alpha}\right) X^{k-j}
$$

Using this in (1.7), transposing the $Y_{\beta}$ factors, and applying the Schwarz inequality, we obtain the estimate

$$
\left\|\partial \pi\left(Y_{j_{1}} \cdots Y_{j_{m}} X^{k}\right) v\right\|^{2} \leqq \sum_{j=0}^{k}\left(\begin{array}{c}
k \\
j
\end{array}\right)\left\{\sum_{|\beta|=2 m}\left|C_{\alpha, \beta}^{j}\right|\right\}\left\|\partial \pi(X)^{2 k-j} v\right\| \tau_{2 m}(v),
$$


where $\alpha=\left(j_{m}, \ldots, j_{1}, j_{1}, \ldots, j_{m}\right)$. Since this holds for any $\left(j_{1}, \ldots, j_{m}\right)$, we thus have the second a priori estimate

$$
\left[\tau_{m}\left(\partial \pi(X)^{k} v\right)\right]^{2} \leqq \sum_{j=0}^{k}\left(\begin{array}{l}
k \\
j
\end{array}\right) a_{2 m, j}\left\|\partial \pi(X)^{2 k-j} v\right\| \tau_{2 m}(v)
$$

holding for all $v \in \mathscr{H}^{\infty}(\pi)$.

By the induction hypothesis there are polynomials $q_{j}(t), 1 \leqq j \leqq d$, so that the theorem is true for the restriction of $\pi$ to the subgroup exp $\mathfrak{h}$. Assume now that $r, s>0$ and that $v \in \mathscr{H}^{\infty}(\pi)$ satisfies the estimates

$$
\begin{aligned}
\left\|\partial \pi\left(Y_{j}\right)^{n} v\right\| & \leqq M q_{j}(r)^{-n} n !, \quad 1 \leqq j \leqq d, \\
\left\|\partial \pi(X)^{n} v\right\| & \leqq M s^{-n} n !
\end{aligned}
$$

for $n=1,2, \ldots$ By the induction hypothesis $v$ will then satisfy an estimate $\tau_{n}(v)$ $\leqq M_{1} r^{-n} n$ !. (We may assume $q_{j}(t)$ is monotone increasing in $t$.) Hence by (1.8) and the inequality $k !(2 k-j) ! \leqq(2 k) !(k-j)$ ! we have

$$
\left[\tau_{m}\left(\partial \pi(X)^{k} v\right)\right]^{2} \leqq M M_{1} r^{-2 m} S^{-2 k}(2 m) !(2 k) ! \sum_{j=0}^{\infty} \frac{s^{j}}{j !} a_{2 m, j}
$$

Using (1.6), we thus obtain the growth estimate

$$
\tau_{m}\left(\partial \pi(X)^{k} v\right) \leqq M_{2} r^{-m} s^{-k}\left[C_{1}\left(1+s^{N}\right)\right]^{m} k ! m !
$$

with a constant $C_{1}$ independent of $\pi$. By the a priori estimate (1.5), this implies that

$$
\rho_{n}(v) \leqq M_{2} n ! \max _{k+m=n}\left\{s^{-k} r^{-m}\left[C_{2}\left(1+s^{2 N}\right)\right]^{m}\right\},
$$

using (1.6) again, with a constant $C_{2}$ independent of $\pi$. In particular, if $\dot{r}$ and $s$ are related by $r=C_{2} s\left(1+s^{2 N}\right)$, and if $v$ satisfies (1.9), then $v$ satisfies $\rho_{n}(v) \leqq M_{2} n ! s^{-n}$.

We conclude from the estimates of the last paragraph that (1.3) holds, with the polynomials $p_{j}(t)$ taken as $q_{j}\left(C t\left(1+t^{2 N}\right)\right)$ for $1 \leqq j \leqq d$, and $p_{d+1}(t)=t$. If the polynomials $q_{j}(t)$ are assumed to be of the form (1.4), then with suitable choice of the constant $C$ one easily verifies that suitable $p_{j}(t)$ can be also given by (1.4), relative to the basis $\left\{X_{j}\right\}_{1 \leqq j \leqq d+1}$. Q.E.D.

COROLlaRy 1.1. Let $\pi$ be a unitary representation of $G$, and let $v \in \mathscr{H}(\pi)$. Suppose that for some $r>0$ and all $j, 1 \leqq j \leqq d$, the function $t \rightarrow\left(\pi\left(\exp t X_{j}\right) v, v\right), t$ real, extends holomorphically to the complex disc $|t|<2 p_{j}(r)$. Then $v \in \mathscr{H}_{r}^{\omega}(\pi)$. In particular, $v$ is an entire vector for $\pi$ if and only if the associated positive-definite function $g \rightarrow(\pi(g) v, v)$ extends holomorphically to an entire function on $G_{c}$.

Proof. Under the stated assumptions on $v$, we conclude from Proposition 4.1 of [5] that $v \in \mathscr{H}_{p_{f}(r)}^{\omega}\left(\pi_{j}\right), 1 \leqq j \leqq d$. Hence by Theorem 1.1 we have $v \in \mathscr{H}_{r}^{\omega}(\pi)$. If this holds for all $r$, then $v$ will be an entire vector. Conversely, if $v$ is an entire vector, then by Corollary 2.1 of [5] the function $X \rightarrow(\pi(\exp X) v, v)$ on $g$ extends holomorphically to $\mathfrak{g}_{c}$. Q.E.D. 
2. Analytic vectors for the regular representation. Let $L$ be the left regular representation of $G$ on $L_{2}(G)$ :

$$
(L(x) f)(y)=f\left(x^{-1} y\right) .
$$

An explicit description of the analytic vectors for $L$ is given by

THEOREM 2.1. Suppose $f \in \mathscr{H}_{t}^{\omega}(L), t>0$. Then $f$ is a $C^{\infty}$ function on $G$, such that

(i) $f$ can be analytically continued to the connected open subset $\Omega_{t} G$ of $G_{c}$, where $\Omega_{t}=\left\{\exp X ; X \in \mathfrak{g}_{c},|X|<t\right\}$.

(ii) If $0<s<t$, then

$$
\sup _{\gamma \in \Omega_{s}} \int_{G}|f(\gamma g)|^{2} d g<\infty .
$$

Conversely, let the polynomials $p_{j}(t)$ be as in Theorem 1.1, and suppose that $f$ is a $C^{\infty}$ function on $G$ such that for fixed $g \in G$, the function $\xi \rightarrow f\left(\exp \left(\xi X_{j}\right) g\right)$, $\xi$ real, can be analytically continued to the complex disc $|\zeta|<p_{j}(t), j=1, \ldots, d$. Assume that the analytic continuations satisfy

$$
\sup _{|\zeta| \leqq s} \int\left|f\left(\exp \left(\zeta X_{j}\right) g\right)\right|^{2} d g<\infty
$$

for $1 \leqq j \leqq d, s<p_{j}(t)$. Then $f \in \mathscr{H}_{t}^{\omega}(L)$.

Proof. Suppose $f \in \mathscr{H}_{t}^{\omega}(L)$. By Sobolev's lemma (cf. Proposition 5.1 of [13]), $\mathscr{H}^{\infty}(L) \subseteq C^{\infty}(G)$ continuously, so that point evaluations on $G$ define continuous linear functionals on $\mathscr{H}^{\infty}(L)$. Hence $f$ is $C^{\infty}$, and by Corollary 2.1 of [5], the function $X \rightarrow f(\exp X g)$ extends holomorphically to the region $|X|<t$ in $g_{c}$, for each $g \in G$, giving (i). By Proposition 2.2 of [5] and the uniqueness of analytic continuation, we have the identity

$$
L_{\omega}(\gamma) f(g)=f\left(\gamma^{-1} g\right)
$$

holding for $\gamma \in \Omega_{t}, g \in G$ ( $L_{\omega}$ denoting the analytic continuation of the representation $L$ to a local representation of $G_{c}$ on $\mathscr{H}^{\omega}(L)$, as defined in [5]). Since $\gamma \rightarrow L_{\omega}(\gamma) f$ is holomorphic from $\Omega_{t}$ to $L_{2}(G)$, it is bounded on compact subsets of $\Omega_{t}$, yielding (ii).

For the converse, we first recall the fact that if $\mathfrak{h}$ is an ideal in $\mathfrak{g}$ of codimension one, and we write $\mathfrak{g}=\mathfrak{h} \oplus(X)$, then the maps

$$
(t, Y) \rightarrow \exp t X \exp Y, \quad(t, Y) \rightarrow \exp Y \exp t X
$$

from $\boldsymbol{R} \times \mathfrak{h}$ to $G$ are analytic manifold isomorphisms, and that a Haar measure on $G$ is given by the product of Lebesgue measures on $\boldsymbol{R}$ and $\mathfrak{h}$ in these coordinates (cf. [14, Part II, Chapter II, §2, Corollary 2, Remark 2]).

As a consequence, we may take as global coordinates on $G$ the "canonical coordinates of the second kind" given by

$$
\Phi_{j}:\left(t_{1}, \ldots, t_{d}\right) \rightarrow g_{j}\left(t_{j}\right) g_{1}\left(t_{1}\right) \cdots\left(g_{j}\left(t_{j}\right)\right)^{\wedge} \cdots g_{d}\left(t_{d}\right),
$$


the circumflex denoting omission. Here $g_{k}(t)=\exp t X_{k}$, and we have picked a particular one-parameter subgroup $g_{j}(t)$ to appear on the left, the other oneparameter subgroups occurring in increasing order from left to right. In these coordinates a Haar measure on $G$ is given by Lebesgue measure on $\boldsymbol{R}^{d}$.

Let $f \in C^{\infty}(G)$ be such that the function $\xi \rightarrow f\left(\exp \left(\xi X_{j}\right) g\right)$ can be analytically continued to the complex disc $|\zeta|<r, r=p_{j}(t)$. Since $\exp (\xi X) \Phi_{j}\left(t_{1}, \ldots, t_{d}\right)$ $=\Phi_{j}\left(t_{1}, \ldots, t_{j}+\xi, \ldots, t_{d}\right)$, this is equivalent to the assumption that the function $F_{j}=f \circ \Phi_{j}$ can be analytically continued in the variable $t_{j}$ to the strip $\left|\operatorname{Im} t_{j}\right|<r$, for fixed real values of the remaining $t_{k}$. By expanding $F_{j}$ in a Taylor series in the variable $t_{j}$, we see that the function $g \rightarrow f\left(\exp \left(\zeta X_{j}\right) g\right)$ is measurable for fixed complex $\zeta,|\zeta|<r$. Hence the integral in condition (2.1) is defined.

Assume now that $f$ can be analytically continued as above, and that condition (2.1) is satisfied. In the coordinate system $\Phi_{j}$ this condition becomes

$$
\sup _{|\eta|<s} \int_{R^{d}}\left|F_{j}\left(t_{1}, \ldots, t_{j}+i \eta, \ldots, t_{d}\right)\right|^{2} d t_{1} \cdots d t_{d}<\infty
$$

for all $s<r$. Let $U_{j}(\xi)$ be the operator of translation by $\xi$ in the $j$ th coordinate, acting on $L_{2}\left(\boldsymbol{R}^{d}\right)$. It follows from Morera's theorem and the Fubini theorem, using (2.4), that the function $\xi \rightarrow U_{j}(\xi) F_{j}$ extends to a holomorphic function from the disc $|\zeta|<r$ to $L_{2}\left(\boldsymbol{R}^{d}\right)$. (We could have invoked the original theorem of Paley and Wiener at this point.) But

$$
U_{j}(\xi) F_{j}=L\left(g_{j}(\xi)\right) f \circ \Phi_{j},
$$

so we deduce that $f \in \mathscr{H}_{p_{j}(t)}^{\omega}\left(L_{j}\right)$. By Theorem 1.1 we obtain $f \in \mathscr{H}_{t}^{\omega}(\pi)$. Q.E.D.

As an immediate consequence of Theorem 2.1 and equation (2.4), we obtain

COROLlaRY 2.1. A function $f$ on $G$ is an entire vector for the left regular representation $L$ if and only if $f$ is the restriction to $G$ of an entire function on $G_{c}$ which satisfies

$$
\sup _{\gamma \in \Omega_{t}} \int_{G}|f(\gamma g)|^{2} d g<\infty
$$

for all $t<\infty$.

The representation $L_{\omega}$ of $G_{c}$ on the space $\mathscr{H}_{\infty}^{\omega}(L)$ is given by left translation.

REMARK. It is an easy consequence of the closed graph theorem (or the explicit estimates of $\S 1$ ) that the family of norms defined by the left side of (2.5), as $t$ ranges over the positive reals, gives the topology on $\mathscr{H}_{\infty}^{\omega}(L)$ defined in [5].

3. Fourier transforms of analytic vectors. In this section we will obtain the characterization of the Fourier transforms of analytic vectors for the left regular representation (Theorem $B$ of the Introduction). We first state and prove two general lemmas concerning analytic vectors for direct integrals and tensor products of unitary representations. 
LEMMA 3.1. Let $U$ be a unitary representation of $G$, and assume that

$$
U=\int^{\oplus} U^{\lambda} d \lambda, \quad \mathscr{H}(U)=\int^{\oplus} \mathscr{H}\left(U^{\lambda}\right) d \lambda
$$

is a direct integral decomposition of $U$. Let the polynomials $p_{j}(t)$ be as in Theorem 1.1, and set $\delta=(\operatorname{dim} G)^{1 / 2}$. The analytic vectors for $U$ decompose as follows:

(a) If $v \in \mathscr{H}_{t}^{\omega}(U)$ for some $t>0$, then the components $v^{\lambda} \in \mathscr{H}_{t}^{\omega}\left(U^{\lambda}\right)$ a.e. $(d \lambda)$, and for all $s<t$,

$$
\int \sup _{\gamma \in \Omega_{s}}\left\|U_{\omega}^{\lambda}(\gamma) v^{\lambda}\right\|^{2} d \lambda<\infty
$$

(b) Conversely, if $v \in \mathscr{H}(U)$ is such that $v^{\lambda} \in \mathscr{H}_{p_{j}(t)}^{\omega}\left(U_{j}^{\lambda}\right)$ and

$$
\int \max _{z= \pm i s}\left\|U_{\omega}^{\lambda}\left(\exp z X_{j}\right) v^{\lambda}\right\|^{2} d \lambda<\infty
$$

for all $s, 0<s<p_{j}(t)$, and $1 \leqq j \leqq d$, then $v \in \mathscr{H}_{t}^{\omega}(U)$.

(c) If $v \in \mathscr{H}_{t}^{\omega}(U)$ and $\gamma \in \Omega_{t}$, then the $\lambda$-component of $U_{\omega}(\gamma) v$ is $U_{\omega}^{\lambda}(\gamma) v^{\lambda}$ a.e. $(d \lambda)$.

Proof. (a) We first establish that the space $\mathscr{H}^{\infty}(U)$ decomposes in the direct integral as the space of sections $\left\{v^{\lambda}\right\}$ such that

$$
v^{\lambda} \in \mathscr{H}^{\infty}\left(\pi^{\lambda}\right) \text { a.e., }
$$

and

$$
\int\left\|\partial U^{\lambda}(W) v^{\lambda}\right\|^{2} d \lambda<\infty
$$

for any $W \in \mathfrak{U}(\mathfrak{g})$.

Indeed, if $X \in \mathrm{g}$ and $\pi$ is a unitary representation of $G$, then by Stone's theorem we may write $\pi(\exp t X)=e^{t d \pi(X)}$, where $d \pi(X)$ is a skew-adjoint operator on $\mathscr{H}(\pi)$. We have the representation formula

$$
[1-d \pi(X)]^{-1}=\int_{0}^{\infty} \pi(\exp t X) e^{-t} d t
$$

relating the resolvent of $d \pi(X)$ and the one-parameter group $\pi(\exp t X)$.

Let $\mathscr{H}^{k}(\pi)$ denote the space of $k$-times differentiable vectors for a representation $\pi$ (cf. [7]), and suppose that $v \in \mathscr{H}^{1}(U)$. Set $w=d U(X) v$. Then by (3.5) we have

Hence

$$
v=\int_{0}^{\infty} U(\exp t X)(v+w) e^{-t} d t
$$

$$
\begin{aligned}
v^{\lambda} & =\int_{0}^{\infty} U^{\lambda}(\exp t X)\left(v^{\lambda}+w^{\lambda}\right) e^{-t} d t \quad \text { a.e. }(d \lambda) \\
& =\left[1-d U^{\lambda}(X)\right]^{-1}\left(v^{\lambda}+w^{\lambda}\right) \quad \text { a.e. }(d \lambda)
\end{aligned}
$$

so that $v^{\lambda} \in \mathscr{D}\left(d U^{\lambda}(X)\right)$ a.e. $(d \lambda)$, and $d U^{\lambda}(X) v^{\lambda}=w^{\lambda}$ a.e. $(d \lambda)$. $(\mathscr{D}(S)$ denotes the domain of definition of an operator $S$.) By Proposition 1.1 of [7] we conclude that 
$v^{\lambda} \in \mathscr{H}^{1}\left(U^{\lambda}\right)$ a.e. $(d \lambda)$. Iterating this argument, we obtain (3.3) and (3.4) for any $v \in \mathscr{H}^{\infty}(U)$, and the equation

$$
(\partial U(W) v)^{\lambda}=\partial U^{\lambda}(W) v^{\lambda} \quad \text { a.e. }(d \lambda), \quad W \in \mathfrak{H}(\mathfrak{g})_{c} .
$$

Conversely, if $v^{\lambda} \in \mathscr{H}^{1}\left(U^{\lambda}\right)$ a.e. $(d \lambda)$, and

$$
\int\left\|d U^{\lambda}(X) v^{\lambda}\right\|^{2} d \lambda<\infty
$$

for all $X \in \mathfrak{g}$, let $w \in \mathscr{H}(U)$ be such that $w^{\lambda}=d U^{\lambda}(X) v^{\lambda}$. Then

$$
U^{\lambda}(\exp t X) v^{\lambda}-v^{\lambda}=\int_{0}^{t} U^{\lambda}(\exp s X) w^{\lambda} d s
$$

so

$$
\left\|U^{\lambda}(\exp t X) v^{\lambda}-v^{\lambda}\right\| \leqq|t|\left\|w^{\lambda}\right\| .
$$

By the dominated convergence theorem, we obtain $v \in \mathscr{D}(d U(X))$. Iteration of this argument (using Proposition 1.1 of [7] again) shows that (3.3) and (3.4) imply that $v \in \mathscr{H}^{\infty}(U)$.

Suppose now that $v \in \mathscr{H}^{\infty}(U)$. Recall the notation $X_{\alpha}=X_{j_{1}} \cdots X_{j_{n}}$, where $\alpha=\left(j_{1}, \ldots, j_{n}\right)$ with $1 \leqq j_{k} \leqq d$ is a multi-index, $|\alpha|=n$, and $\left\{X_{j}\right\}$ is the fixed JordanHölder basis for $g$. If $r>0$, then by the monotone convergence theorem and equation (3.6) we have

$$
\sum_{n=0}^{\infty} \frac{r^{2 n}}{(n !)^{2}} \sum_{|\alpha|=n}\left\|\partial U\left(X_{\alpha}\right) v\right\|^{2}=\int \sum_{n=0}^{\infty} \frac{r^{2 n}}{(n !)^{2}} \sum_{|\alpha|=n}\left\|\partial U^{\lambda}\left(X_{\alpha}\right) v^{\lambda}\right\|^{2} d \lambda .
$$

Since there are $d^{n}$ multi-indices of length $n$, the left side of (3.7) is bounded by $\sum\left(r^{2} d\right)^{n}(n !)^{-2} \rho_{n}(v)^{2}$, where $\left\{\rho_{n}\right\}$ are the canonical seminorms on $\mathscr{H}^{\infty}(U)$, as in $\S 1$. Hence if $v \in \mathscr{H}_{t \sqrt{ } d}^{\omega}(U)$, then the left side of (3.7) is finite when $r<t$. It follows in this case that the integrand on the right side is finite a.e. Since the integrand dominates $\sum r^{2 n}(n !)^{-2} \rho_{n}^{\lambda}\left(v^{\lambda}\right)^{2}$, we conclude that $v^{\lambda} \in \mathscr{H}_{t}^{\omega}\left(U^{\lambda}\right)$ a.e. (Here $\rho_{n}^{\lambda}$ denotes the canonical seminorm on $\mathscr{H}^{\infty}\left(U^{\lambda}\right)$.) From the power series definition of $U_{\omega}^{\lambda}(\gamma)$ we also have from (3.7)

$$
\sup _{\gamma \in \Omega_{s}}\left\|U_{\omega}^{\lambda}(\gamma) v^{\lambda}\right\| \leqq \Phi_{s}(\lambda)
$$

where $\Phi_{s} \in L_{2}(d \lambda)$.

Since $\left\|U_{\omega}^{\lambda}(\gamma) v^{\lambda}\right\|$ is continuous in $\gamma$, the supremum in (3.8) is obtained by letting $\gamma$ run over a fixed countable dense subset of $\Omega_{s}$, and hence is a measurable function of $\lambda$. This proves part (a) of the lemma.

Let $v$ satisfy the conditions of part (b) of the lemma. We observe that by the maximum modulus principle and the unitarity of $U$, we have

$$
\sup _{\mid z ! \leqq r}\left\|U_{\omega}^{\lambda}\left(\exp z X_{j}\right) v^{\lambda}\right\|=\max _{z= \pm i r}\left\|U_{\omega}^{\lambda}\left(\exp z X_{j}\right) v^{\lambda}\right\| .
$$


Hence by the Cauchy estimates and (3.7), $v \in \mathscr{H}_{p_{j}(t)}^{\omega}\left(U_{j}\right)$. Thus $v \in \mathscr{H}_{t}^{\omega}(U)$ by Theorem 1.1.

To prove (c), we start with the formula

$$
(U(\gamma) v, w)=\int\left(U^{\lambda}(\gamma) v^{\lambda}, w^{\lambda}\right) d \lambda
$$

valid for $\gamma \in G$ and $v, w \in \mathscr{H}(U)$. If $v \in \mathscr{H}_{\delta t}^{\omega}(U)$, then by part (a), using the theorems of Morera and Fubini, we see that the right side of this equation has an anatic continuation to the region $\gamma \in \Omega_{t}$ given by $\int\left(U_{\omega}^{\lambda}(\gamma) v^{\lambda}, w^{\lambda}\right) d \lambda$. By Proposition 2.2 of [5], the left side has the analytic continuation $\left(U_{\omega}(\gamma) v, w\right)$. Since $\Omega_{t}$ is connected, these two continuations must coincide, and hence (c) follows by the arbitrariness of $w$. Q.E.D.

LEMMA 3.2. Let $\pi$ be a unitary representation of $G$, and let $\Lambda$ be the representation given by left multiplication by $\pi(g)$ acting on the space of Hilbert-Schmidt operators on $\mathscr{H}(\pi)$. Let the polynomials $p_{j}(t)$ be as in Theorem 1.1. Then the analytic vectors for $\Lambda$ may be described as follows:

(a) If $T \in \mathscr{H}_{t}^{\omega}(\Lambda)$, then Range $(T) \subseteq \mathscr{H}_{t}^{\omega}(\pi)$, and for all $s<t$,

$$
\sup _{\gamma \in \Omega_{s}}\left\|\pi_{\omega}(\gamma) T\right\|_{2}<\infty
$$

$\left(\|\cdot\|_{2}\right.$ denoting the Hilbert-Schmidt operator norm).

(b) Conversely, if $T$ is a Hilbert-Schmidt operator on $\mathscr{H}(\pi)$ such that Range $(T)$ $\subseteq \mathscr{H}_{p_{j}(t)}^{\omega}\left(\pi_{j}\right)$, and if $\pi_{\omega}\left(\exp \pm\right.$ is $\left.X_{j}\right) T$ is a Hilbert-Schmidt operator for $0<s<p_{j}(t)$, $1 \leqq j \leqq d$, then $T \in \mathscr{H}_{t}^{\omega}(\Lambda)$.

(c) The analytic continuation of $\Lambda$ is given by left multiplication by $\pi_{\omega}(\gamma)$.

Proof. (a) We first note that the $C^{\infty}$ vectors for $\Lambda$ consist of all Hilbert-Schmidt operators $T$ on $\mathscr{H}(\pi)$ such that

(i) Range $(T) \subseteq \mathscr{H}^{\infty}(\pi)$,

(ii) $\partial \pi(W) T$ is a Hilbert-Schmidt operator for all $W \in \mathfrak{u}(\mathfrak{g})_{c}$.

Indeed, if $\mathscr{H}^{k}(\pi)$ and $\mathscr{H}^{k}(\Lambda), k=0,1,2, \ldots$, denote the chains of spaces of $k$-times differentiable vectors for $\pi$ and $\Lambda$ respectively (cf. [7]), then for $T \in \mathscr{H}^{1}(\Lambda)$ and $X \in \mathfrak{g}$ we may write

$$
\pi(\exp t X) T=T+\int_{0}^{t} \pi(\exp s X) T_{1} d s,
$$

where $T_{1}=\partial \Lambda(X) T$ is a Hilbert-Schmidt operator, and the integral in (3.10) converges in the Hilbert-Schmidt operator norm. Hence if $v \in \mathscr{H}(\pi)$, then

$$
[\pi(\exp t X)-1] T v=\int_{0}^{t} \pi(\exp s X) T_{1} v d s,
$$

so that $T v \in \mathscr{D}(d \pi(X))$ and $d \pi(X) T v=T_{1} v$. Since this holds for all $X \in \mathfrak{g}$, we have Range $(T) \subseteq \mathscr{H}^{1}(\pi)$ and $d \pi(X) T=d \Lambda(X) T$. Conversely, if Range $(T) \subseteq \mathscr{H}^{1}(\pi)$ and 
$d \pi(X) T=T_{1}$, then the integral formula (3.10) is valid, where the integral converges in the strong operator topology. If $T_{1}$ is again a Hilbert-Schmidt operator, then the integral converges in the Hilbert-Schmidt norm, so that $T \in \mathscr{H}^{1}(\Lambda)$. Iteration of this argument and the use of Proposition 1.1 of [7] establishes that $T \in \mathscr{H}^{k}(\Lambda)$ if and only if Range $(T) \subseteq \mathscr{H}^{k}(\pi)$ and $d \pi\left(Y_{1}\right) \cdots d \pi\left(Y_{k}\right) T$ is a Hilbert-Schmidt operator for any $Y_{1}, \ldots, Y_{k} \in \mathfrak{g}$. In particular, we obtain (i) and (ii). (In case $\pi$ is irreducible, the chain $\left\{\mathscr{H}^{k}(\pi)\right\}$ is nuclear, and one may show that (i) implies (ii). We shall not need this fact in the following however.) The proof just given also shows that $\partial \Lambda(W)$ is simply left multiplication by $\partial \pi(W)$, for $W \in \mathfrak{H}(\mathfrak{g})_{c}$.

Let $\rho_{n}^{\pi}$ and $\rho_{n}^{\Lambda}$ be the seminorms on $\mathscr{H}^{\infty}(\pi)$ and $\mathscr{H}^{\infty}(\Lambda)$ respectively, as defined in $\S 1$. If $T \in \mathscr{H}^{\infty}(\Lambda)$ and $v \in \mathscr{H}(\pi)$, we have by (i) and (ii) above the a priori estimate

$$
\rho_{n}^{\pi}(T v) \leqq \rho_{n}^{\Lambda}(T)\|v\| .
$$

Suppose now that $T \in \mathscr{H}_{t}^{\omega}(\Lambda)$. Then (3.11) implies that $T v \in \mathscr{H}_{t}^{\omega}(\pi)$ for all $v \in \mathscr{H}_{(\pi)}$. Thus the operator $\pi_{\omega}(\gamma) T$ is holomorphic in $\gamma \in \Omega_{t}$, and agrees with $\Lambda_{\omega}(\gamma) T$ when $\gamma \in G$. By the uniqueness of analytic continuation, we have $\pi_{\omega}(\gamma) T=\Lambda_{\omega}(\gamma) T$, when $\gamma \in \Omega_{t}$. Hence (3.9) follows from Proposition 2.2 of [5].

(b) Suppose $T$ is a Hilbert-Schmidt operator on $\mathscr{H}(\pi)$ satisfying the conditions of part (b) of Lemma 3.2. If $\zeta=\xi+i s$, with $\xi$, $s$ real and $|\zeta|<p_{j}(t)$, then

$$
\pi_{\omega}\left(\exp \zeta X_{j}\right) T=\pi\left(\exp \xi X_{j}\right) \pi_{\omega}\left(\exp i s X_{j}\right) T \text {. }
$$

Since $\pi\left(\exp \xi X_{j}\right)$ is unitary, it follows that the operator $\pi_{\omega}\left(\exp \zeta X_{j}\right) T$ is HilbertSchmidt, and

$$
\left\|\pi_{\omega}\left(\exp \zeta X_{j}\right) T\right\|_{2}=\left\|\pi_{\omega}\left(\exp i s X_{j}\right) T\right\|_{2} .
$$

Since $\pi_{\omega}\left(\exp \zeta X_{j}\right) \subseteq e^{\zeta d \pi\left(X_{j}\right)}$, it follows from the spectral theorem that if $r$ is real and $|r|<s$, then

$$
\left\|\pi_{\omega}\left(\exp i r X_{j}\right) v\right\| \leqq \max _{\zeta= \pm i s}\left\|\pi_{\omega}\left(\exp \zeta X_{j}\right) v\right\| .
$$

Combining these two estimates, we conclude that $\sup _{|\zeta|=s}\left\|\pi_{\omega}\left(\exp \zeta X_{j}\right) T\right\|_{2}$ is a nondecreasing finite-valued function of $s, s<p_{j}(t)$. By the usual argument, via Fubini's theorem and Morera's theorem, we obtain the analyticity of the function

$$
\zeta \rightarrow \operatorname{tr}\left(\pi_{\omega}\left(\exp \zeta X_{j}\right) T S^{*}\right)
$$

for $|\zeta|<p_{j}(t)$ and $S$ an arbitrary Hilbert-Schmidt operator. Theorem 1.1 then gives $T \in \mathscr{H}_{t}^{\omega}(\Lambda)$. Q.E.D.

We now give the dual version of Theorem 2.1. For this, let $\hat{G}, \mu, \pi^{\xi}$ be as in the Introduction, let $p_{j}(t)$ be the polynomials in Theorem 1.1 and set $\delta=(\operatorname{dim} G)^{1 / 2}$.

THEOREM 3.1. Let $f \in L_{2}(G)$, and let $\hat{f}$ be its Fourier transform. Suppose $f \in \mathscr{H}_{\delta t}^{\omega}(L)$ for some $t>0$. Then

(i) Range $\hat{f}(\xi) \subseteq \mathscr{H}_{t}^{(i)}\left(\pi^{\xi}\right)$ a.e. $(\mu)$;

(ii) $\left(L_{\omega}(\gamma) f\right)^{\wedge}(\xi)=\pi_{\omega}^{\xi}(\gamma) \hat{f}(\xi)$ a.e. $(\mu)$ when $\gamma \in \Omega_{t}$;

(iii) $\int_{\hat{G}} \sup _{\gamma \in \Omega_{s}}\left\|\pi_{\omega}^{\xi}(\gamma) \hat{f}(\xi)\right\|_{2}^{2} d \mu(\xi)<\infty$ for all $s<t$. 
Conversely, if $\xi \rightarrow \hat{f}(\xi)$ is a $\mu$-measurable field of operators such that for $1 \leqq j \leqq d$ and $0<s<p_{j}(t)$ one has

$$
\begin{gathered}
\text { Range } \hat{f}(\xi) \subseteq \mathscr{H}_{p_{j}(t)}^{\omega}\left(\pi_{j}^{\xi}\right) \text { a.e. }(\mu), \\
\int_{\hat{G}} \max _{\zeta= \pm i s}\left\|\pi_{\omega}^{\xi}\left(\exp \zeta X_{j}\right) \hat{f}(\xi)\right\|_{2}^{2} d \mu(\xi)<\infty
\end{gathered}
$$

then $\hat{f}$ is the Fourier transform of a function $f \in \mathscr{H}_{t}^{\omega}(L)$, and $\hat{f}$ satisfies (i), (ii), and (iii).

REMARK. It was shown in the proof of Lemma 3.2 that the integrand in (3.13) is a nondecreasing function of $s$. Hence to check that $\hat{f}$ is the Fourier transform of an analytic vector for $L$, it suffices to have (3.12) satisfied for some $t>0$, and (3.13) satisfied for some positive $s<\min _{j} p_{j}(t)$, for all $j, 1 \leqq j \leqq d$.

Proof of Theorem 3.1. By the Plancherel theorem [3], $L=\iint^{\oplus} \Lambda^{\xi} d \mu(\xi)$, where $\Lambda^{\xi}$ is the representation given by left multiplication by $\pi^{\xi}$ on the space of HilbertSchmidt operators. The theorem thus follows immediately from Lemmas 3.1 and 3.2. Q.E.D.

As an immediate consequence of Theorem 3.1 we have

CoRollary 3.1. A function $f$ on $G$ is an entire vector for the left regular representation $L$ if and only if its Fourier transform $\hat{f}$ satisfies

(i) Range $\hat{f}(\xi)$ is contained in the space of entire vectors for $\pi^{\xi}$ a.e. $(\mu)$.

(ii) For every $t>0$,

$$
\int_{\hat{G}} \sup _{\gamma \in \Omega_{t}}\left\|\pi_{\omega}^{\xi}(\gamma) \hat{f}(\xi)\right\|_{2}^{2} d \mu(\xi)<\infty .
$$

The Fourier transform of the representation $L_{\omega}$ is given by left multiplication by $\pi_{\omega}^{\xi}$.

REMARK. The family of norms defined by (3.14), as $t$ ranges over the positive reals, gives the topology on $\mathscr{H}_{\infty}^{\omega}(\hat{L})$ defined abstractly in [5].

4. Analytic continuation of the Fourier inversion formula. Let $f$ be an analytic vector for the left regular representation $L$. In this section we use the results of $\$ 3$ to show that the analytic continuation of $f$, which exists by Theorem 2.1 , is explicitly given by analytic continuation of the Fourier inversion formula. Let $\delta=(\operatorname{dim} G)^{1 / 2}$. If $T$ is an operator on Hilbert space, let $\|T\|_{1}$ denote the trace norm of $T$, and let $\hat{G}, \mu, \pi^{\xi}$ be as in the Introduction.

THEOREM 4.1. Let $f \in \mathscr{H}_{2 \delta t}^{\omega}(L)$. Then for any $s<t$,

$$
\int_{\hat{G}} \sup _{\gamma \in \Omega_{s}}\left\|\pi_{\omega}^{\xi}(\gamma) \hat{f}(\xi)\right\|_{1} d \mu(\xi)<\infty .
$$

The analytic continuation of $f$ to the set $\Omega_{t} G$ is given by the absolutely convergent integral

$$
f(\gamma g)=\int_{\hat{G}} \operatorname{tr}\left(\pi_{\omega}^{\xi}\left(\gamma^{-1}\right) \hat{f}(\xi) \pi^{\xi}\left(g^{-1}\right)\right) d \mu(\xi), \quad \gamma \in \Omega_{t}, g \in G
$$


Proof. Let $\Delta=\sum_{j=1}^{d} X_{j}^{2}$. If $\pi$ is a unitary representation of $G$, we denote by $A(\pi)$ the selfadjoint operator $\partial \pi(1-\Delta)^{*}$, and by $B(\pi)$ the positive square root of $A(\pi)$. When $\pi=L$ is the left regular representation, we will simply write $A$ and $B$ respectively. Let $t>0$. Then for any $T \in \mathfrak{u}(\mathfrak{g})$,

$$
\text { Range }\left(\partial L(T) e^{-t A}\right) \subseteq \bigcap_{n} \mathscr{D}\left(A^{n}\right)=\mathscr{H}^{\infty}(L)
$$

[10]. Hence by a Sobolev lemma (Proposition 5.1 of [13]), $\partial L(T) e^{-t A}$ maps $L_{2}(G)$ continuously into $C^{\infty}(G)$. Since it commutes with right translations, it follows that $e^{-t A}$ is given by left convolution with an $L_{2}$ function $K_{t}$, and that $g, t \rightarrow K_{t}(g)$ is $C^{\infty}$ (cf. [15]). Furthermore, Nelson and Stinespring [11] proved that $K_{t} \in L_{1}(G)$, $\left\|K_{t}\right\|_{L_{1}(G)} \leqq 1$, and that for an arbitrary unitary representation $\pi$,

$$
e^{-t A(\pi)}=\int_{G} \pi(g) K_{t}(g) d g
$$

The semigroup $t \rightarrow e^{-t B(\pi)}$ can be obtained from the semigroup $t \rightarrow e^{-t A(\pi)}$ by an integral formula [16, Chapter IX, §11]. Using this formula, (4.3), and the Fubini theorem, we conclude that

$$
e^{-t B(\pi)}=\int_{G} \pi(g) W_{t}(g) d g
$$

where

$$
W_{t}(g)=c t \int_{0}^{\infty} K_{s}(g) e^{-t^{2} / 4 s} s^{-3 / 2} d s
$$

$c=(2 \sqrt{ } \pi)^{-1}$. If $\hat{W}_{t}$ is the $L_{2}$-Fourier transform of $W_{t}$, we thus have by $(0.1)$ and the above the identity

$$
\hat{W}_{t}(\xi)=e^{-t B\left(\pi^{\xi}\right)} \quad \text { a.e. }(\mu) .
$$

We next observe, that if $v \in \mathscr{H}^{\infty}(\pi)$, then

$$
\left\|B(\pi)^{n} v\right\|^{2}=\left(\partial \pi(1-\Delta)^{n} v, v\right) \leqq \sum_{k=0}^{n}\left(\begin{array}{l}
n \\
k
\end{array}\right) d^{k} \rho_{k}(v)^{2} .
$$

Here $\left\{\rho_{k}\right\}$ are the canonical seminorms on $\mathscr{H}^{\infty}(\pi)$, and we have used the obvious estimate

$$
(-1)^{k}\left(\partial \pi(\Delta)^{k} v, v\right) \leqq d^{k} \rho_{k}(v)^{2} .
$$

Hence if the norms $M_{s}^{\pi}$ on $\mathscr{H}_{t}^{\omega}(\pi)$ are defined by (1.1), $s<t$, then for $v \in \mathscr{H}_{t}^{\omega}(\pi)$ and $\alpha$ a multi-index of length $m$, we have

$$
\begin{aligned}
\left\|B(\pi)^{n} \partial \pi\left(X_{\alpha}\right) v\right\|^{2} & \leqq M_{s}^{\pi}(v)^{2} \sum_{k=0}^{n}\left(\begin{array}{l}
n \\
k
\end{array}\right) d^{k} s^{-2(k+m)}[(k+m) !]^{2} \\
& \leqq M_{s}^{\pi}(v)^{2} 4^{m} s^{-2 m}\left(1+4 d s^{-2}\right)^{n}(m ! n !)^{2}
\end{aligned}
$$


Thus if $X \in \mathfrak{g}_{c}$, then

$$
\left\|B(\pi)^{n} \partial \pi(X)^{m} v\right\| \leqq M_{s}^{\pi}(v)\left(2 s^{-1}|X|\right)^{m} c(s)^{n} m ! n !,
$$

where $c(s)=\left(1+4 d s^{-2}\right)^{1 / 2}$. Let $0<\varepsilon<c(s)^{-1}$, and $|X|<s / 2$. Then from (4.6) we have

$$
\left\|e^{\varepsilon B(\pi)} \pi_{\omega}(\exp X) v\right\| \leqq C M_{s}^{\pi}(v),
$$

where $C=\left(1-2 s^{-1}|X|\right)^{-1}(1-\varepsilon c(s))^{-1}$ is independent of $\pi$.

We can now complete the proof of Theorem 4.1. Let $f \in \mathscr{H}_{2 \delta t}^{\omega}(L)$, and denote by $\Lambda^{\xi}$ the representation given by left multiplication by $\pi^{\xi}$, acting on the space of Hilbert-Schmidt operators on $\mathscr{H}\left(\pi^{\xi}\right)$. By equation (3.7) we have an estimate

$$
M_{s}^{\Lambda^{\xi}}(\hat{f}(\xi)) \leqq \Phi_{s}(\xi),
$$

where $\Phi_{s} \in L_{2}(\hat{G}, d \mu), s<2 t$. From the characterization of the space $\mathscr{H}^{\infty}\left(\Lambda^{\xi}\right)$ given in the proof of Lemma 3.2, we see that the operator $B\left(\Lambda^{\xi}\right)$ is left-multiplication by $B\left(\pi^{\xi}\right)$. Given now $s<t$ and $\gamma \in \Omega_{s}$, choose $s_{1}$ with $2 s<s_{1}<2 t$, and $\varepsilon<c\left(s_{1}\right)^{-1}$. Write

$$
\pi_{\omega}^{\xi}(\gamma) \hat{f}(\xi)=\hat{W}_{\varepsilon}(\xi) e^{\varepsilon B\left(\pi^{\xi}\right)} \pi_{\omega}^{\xi}(\gamma) \hat{f}(\xi) .
$$

Then by the Hölder inequality for the trace-norm and (4.7), (4.8), we have the estimate

$$
\sup _{\gamma \in \Omega_{s}}\left\|\pi_{\omega}^{\xi}(\gamma) \hat{f}(\xi)\right\|_{1} \leqq C \Phi_{s_{1}}(\xi) \hat{W}_{\varepsilon}(\xi)
$$

with the constant $C$ depending only on $s, s_{1}, \varepsilon$. Since $W_{\varepsilon} \in L_{2}(G), \hat{W}_{\varepsilon} \in L_{2}(\hat{G}, d \mu)$ by the Plancherel theorem. Hence the right side of (4.9) is $\mu$-integrable. The left side of (4.9) is easily seen to be $\mu$-measurable, so we obtain (4.1).

To establish the identity (4.2), we observe that the left side is holomorphic for $\gamma \in \Omega_{t}$, by Theorem 2.1. The integrand on the right side is holomorphic for $\gamma \in \Omega_{t}$ and $\mu$-almost all $\xi$, by (4.1) and Theorem 3.1, while another use of (4.1) allows us to apply the Fubini theorem and Morera's theorem to obtain the analyticity of the right side of (4.2). By the Fourier inversion formula both sides agree when $\gamma \in G$, hence by the uniqueness of analytic continuation (4.2) holds for $\gamma \in \Omega_{t}$ also. Q.E.D.

COROLLARY 4.1. Let $f$ be an entire vector for the left regular representation. Then the analytic continuation of $f$ to $G_{c}$ is given by the absolutely convergent integral

$$
f(\gamma)=\int_{\hat{G}} \operatorname{tr}\left(\pi_{\omega}^{\xi}\left(\gamma^{-1}\right) \hat{f}(\xi)\right) d \mu(\xi) .
$$

EXAMPLE. Let $G$ be the Heisenberg group, consisting of all matrices

$$
\left[\begin{array}{lll}
1 & a & c \\
0 & 1 & b \\
0 & 0 & 1
\end{array}\right]
$$


with $a, b, c$ real. Then $G_{c}$ consists of all such matrices with $a, b, c$ complex. The representations $\left\{\pi^{\lambda}\right\}$ which enter into the Plancherel formula, and the spaces $\mathscr{H}_{t}^{\omega}\left(\pi^{\lambda}\right)$, were described in Theorem 6.2 of [5], where $\lambda \in \boldsymbol{R}-\{0\}$. (The statement of this theorem has a misprint, and should read $\mathscr{H}_{t}^{\omega}\left(\pi^{\lambda}\right) \supseteq \mathscr{A}_{t, \lambda} \supseteq \mathscr{H}_{2 t}^{\omega}\left(\pi^{\lambda}\right)$.) In this parametrization the Plancherel measure is $|\lambda| d \lambda, d \lambda$ denoting Lebesgue measure on $\boldsymbol{R}$ [2]. The space of entire vectors for $\pi^{\lambda}$ is independent of $\lambda$, and consists of all entire functions $f$ on $C$ such that $\sup _{|\operatorname{Im}(z)| \leqq r} e^{r|z|}|f(z)|<\infty$ for all $r>0$.

Let $X, Y, Z$ be the usual basis for $\mathfrak{g}$, satisfying $[X, Y]=Z, Z$ central, as in $[5, \S 6]$, and let $\Delta=X^{2}+Y^{2}+Z^{2}$. The eigenfunctions $\left\{\varphi_{n}^{\lambda}\right\}$ of $\partial \pi^{\lambda}(\Delta)$ are entire vectors for $\pi^{\lambda}[5$, Corollary 6.1]. Explicitly,

$$
\varphi_{n}^{\lambda}(x)=|\lambda|^{1 / 4} \varphi_{n}\left(|\lambda|^{1 / 2} x\right),
$$

where $\varphi_{n}$ is the $n$th Hermite function. Using these functions and Theorem 3.1, we can construct the Fourier transforms of some entire vectors for the left regular representation $L$ of $G$ as follows:

Let $P_{m n}(\lambda)$ be the operator on $L_{2}(R)$ which maps $\varphi_{m}^{\lambda}$ to $\varphi_{n}^{\lambda}$, and sends $\varphi_{k}^{\lambda}$ to 0 , $k \neq m$. Then Range $\left(P_{m n}(\lambda)\right) \subseteq \mathscr{H}_{\infty}^{\omega}\left(\pi^{\lambda}\right)$, and

$$
\left\|\pi_{\omega}^{\lambda}(\gamma) P_{m n}(\lambda)\right\|_{2}^{2}=\left\|\pi_{\omega}^{\lambda}(\gamma) \varphi_{n}^{\lambda}\right\|^{2}
$$

This may be evaluated, using formulas for translates of Hermite polynomials $[9, \S 5.6 .4]$, with the result that if $\gamma$ is given by (4.11), then

$$
\left\|\pi_{\omega}^{\lambda}(\gamma) \varphi_{n}^{\lambda}\right\|^{2}=Q_{n}(a, b, \lambda) \exp \left[-2 \lambda \operatorname{Im}(c)+|\lambda|(\operatorname{Im} a+\operatorname{Im} b)^{2}\right],
$$

where $Q_{n}$ is a polynomial in $a, b$, and $\lambda$.

If $h \in L^{2}(R ;|\lambda| d \lambda)$ and vanishes for large $|\lambda|$, it follows from Corollary 3.1 and (4.12) that the operator-valued function $\hat{f}(\lambda)=h(\lambda) P_{m n}(\lambda)$ is the Fourier transform of an $L_{2}$ function $f$ on $G$ which is an entire vector for $L$. The entire function $f$ may be represented by the Fourier inversion formula

$$
\begin{aligned}
f(\gamma) & =\int_{-\infty}^{\infty} \operatorname{tr}\left(\pi_{\omega}^{\lambda}(\gamma) P_{m n}(\lambda)\right) h(\lambda)|\lambda| d \lambda \\
& =\int_{-\infty}^{\infty}\left(\pi_{\omega}^{\lambda}(\gamma) \varphi_{n}^{\lambda}, \varphi_{m}^{\lambda}\right) h(\lambda)|\lambda| d \lambda .
\end{aligned}
$$

Using the Plancherel formula and the completeness of the eigenfunctions $\left\{\varphi_{n}^{\lambda}\right\}$, we conclude that the entire vectors $f$ thus obtained have a dense span in $L_{2}(G)$. We do not know if a similar construction of entire vectors via Fourier transforms and the operator $\Delta$ is possible on an arbitrary simply-connected nilpotent group. In the next section we instead give a direct construction of entire vectors, without the use of Fourier transforms and irreducible representations.

5. Entire vectors for the regular representation. In this section we use Theorem 2.1 to derive sufficient conditions on an entire function $f$ on $G_{c}$, so that $f$ will be an entire vector for the left regular representation of $G$. 
THEOREM 5.1. There exist numbers $p_{j}>1$ with the following property:

If $F$ is an entire function on $C^{d}$ satisfying an estimate

$$
\left|F\left(z_{1}, \ldots, z_{d}\right)\right| \leqq C \exp \left\{-A \sum_{j=1}^{d}\left|\operatorname{Re} z_{j}\right|^{p_{j}}+B \sum_{j=1}^{d}\left|\operatorname{Im} z_{j}\right|^{p_{j}}\right\},
$$

with $A, B, C$ positive constants, then the function $f$ on $G$ defined by

$$
f\left(\exp t_{1} X_{1} \cdots \exp t_{d} X_{d}\right)=F\left(t_{1}, \ldots, t_{d}\right)
$$

is an entire vector for the left regular representation.

REMARK. The proof of Theorem 5.1 gives $p_{j+1}>m p_{j}$ as a sufficient condition on the exponents $p_{j}$ for the validity of the theorem, where $m$ is an integer determined by the Lie algebra $g$ ( $m=0$ if $g$ is abelian).

As the first step in proving Theorem 5.1, we derive a formula for left translation by $\exp z X_{j}$ in canonical coordinates:

Lemma 5.1. Let $\left\{X_{j}\right\}$ be an ordered Jordan-Hölder basis for $\mathfrak{g}$, and set $g_{j}(t)$ $=\exp t X_{j}$. If $g=g_{1}\left(t_{1}\right) \cdots g_{d}\left(t_{d}\right)$, then $g_{j}(z) g=g_{1}\left(s_{1}\right) \cdots g_{d}(s)$, where

$$
\begin{aligned}
s_{k} & =t_{k}+P_{k j}\left(z, t_{k+1}, \ldots, t_{j-1}\right), \quad k<j-1, \\
s_{j-1} & =t_{j-1}, \\
s_{j} & =t_{j}+z, \\
s_{k} & =t_{k}, \quad k>j .
\end{aligned}
$$

The functions $P_{k j}$ in (5.2) are polynomials.

Proof of Lemma 5.1. By induction on $d$ it suffices to treat the case $j=d$. Let $D=\operatorname{ad} X_{d}$. Then we can write

$$
g_{d}(z) g=\exp \left(t_{1} e^{z D} X_{1}\right) \cdots \exp \left(t_{d-1} e^{z D} X_{d-1}\right) g_{d}\left(t_{d}+z\right) .
$$

Now $e^{z D} X_{k}=X_{k} \bmod \left(\mathfrak{h}_{k-1}\right)$, where $\mathfrak{h}_{k}=\operatorname{span}\left\{X_{l}\right\}_{l \leqq k}$. It follows from the CampbellHausdorff formula [8] that

$$
\exp \left(t_{d-1} e^{z D} X_{d-1}\right)=\exp \left(Y_{1}\right) \exp \left(t_{d-1} X_{d-1}\right)
$$

where $Y_{1} \in \mathfrak{h}_{d-2}$ and is a polynomial function of $z$ and $t_{d-1}$. Hence $s_{d-1}=t_{d-1}$. Furthermore, by the Campbell-Hausdorff formula again, we have

$$
\exp \left(t_{d-2} e^{z D} X_{d-2}\right) \exp Y_{1}=\exp \left\{\left[t_{d-2}+P\left(z, t_{d-1}\right)\right] X_{d-2}+Y_{2}\right\},
$$

where $P$ is a polynomial and $Y_{2} \in \mathfrak{h}_{d-3}$ is a polynomial function of $s, t_{d-1}, t_{d-2}$. Another application of the Campbell-Hausdorff formula gives the desired expression for $s_{d-2}$. Iterating this argument, we obtain formulas (5.2). Q.E.D.

Proof of Theorem 5.1. Let $p_{k}>1$, with $p_{k+1} \geqq p_{k}$, be as yet unspecified, and suppose $F$ and $f$ are as in the theorem, with $F$ satisfying (5.1). If $g=g_{1}\left(t_{1}\right) \cdots g_{d}\left(t_{d}\right)$, 
then by Lemma 5.1 we have $f\left(g_{j}(z) g\right)=F\left(s_{1}, \ldots, s_{d}\right)$, with $s_{k}$ given by formulas (5.2). Now if $k<j$ and $t_{l}$ real, then

$$
\begin{aligned}
& \left|\operatorname{Im} s_{k}\right| \leqq C\left(1+\sum_{k<l<j}\left|t_{l}\right|^{m}+|z|^{n}\right) \\
& \left|\operatorname{Re} s_{k}\right| \geqq\left|t_{k}\right|-C\left(1+\sum_{k<l<j}\left|t_{l}\right|^{m}+|z|^{n}\right),
\end{aligned}
$$

where $m$ and $n$ are positive integers determined by the degrees of the polynomials $P_{j k}$, and $C$ is a constant. Hence from (5.1) and (5.3) we obtain the estimate $\left(p_{0}=0\right)$ :

$$
\left|f\left(g_{j}(z) g\right)\right| \leqq C_{1} \exp \left\{-A \sum_{k}\left|t_{k}\right|^{p_{k}}+B_{1} \sum_{k}\left|t_{k}\right|^{m p_{k-1}}+C_{1}|z|^{q}\right\},
$$

where $q=n p_{d}$.

If we initially pick $p_{k}$ so that $p_{k}>m p_{k-1}$, then by (5.4) the entire function $f$ will satisfy the $L_{2}$ boundedness condition (2.1) for all positive $s$. Hence by Theorem 2.1, $f$ will be an entire vector for the representation $L$. Q.E.D.

COROLlaRY 5.1. The space of entire vectors for the left regular representation is dense in $L_{2}(G)$.

Proof. By the work of Gel'fand and Šilov [4], the restrictions to $\boldsymbol{R}^{d}$ of entire functions satisfying a growth estimate (5.1) (with $A, B, C$ depending on $f, p_{j}>1$ fixed) form a dense subspace of $L_{2}\left(\boldsymbol{R}^{d}\right)$. Since Haar measure on $G$ becomes Lebesgue measure on $\boldsymbol{R}^{d}$ in canonical coordinates, the corollary follows from Theorem 5.1. Q.E.D.

\section{REFERENCES}

1. J. Dixmier, Sur les représentations unitaires des groupes de Lie nilpotents. I, Amer. J. Math. 81 (1959), 160-170. MR 21 \#2705.

2. - Sur les représentations unitaires des groupes de Lie nilpotents. IV, Canad. J. Math. 11 (1959), 321-344. MR 21 \#5693.

3. - Les $C^{*}$-algèbres et leurs représentations, Cahiers Scientifiques, fasc. 29, GauthierVillars, Paris, 1964. MR 30 \#1404.

4. I. M. Gel'fand and G. E. Silov, Generalized functions. Vol. 2: Spaces of fundamental functions, Fizmatgiz, Moscow, 1958; English transl., Academic Press; Gordon and Breach, New York, 1968. MR 21 \#5142a; MR 37 \#5693.

5. R. Goodman, Analytic and entire vectors for representations of Lie groups, Trans. Amer. Math. Soc. 143 (1969), 55-76. MR 40 \#1537.

6. - Differential operators of infinite order on a Lie group. I, J. Math. Mech. 19 (1969/70), 879-894. MR 41 \#396.

7. - One-parameter groups generated by operators in an enveloping algebra, J. Functional Analysis 6 (1970), 218-236.

8. G. Hochschild, The structure of Lie groups, Holden-Day, San Francisco, Calif., 1965. MR 34 \#7696.

9. W. Magnus, F. Oberhettinger and R. P. Soni, Formulas and theorems for the special functions of mathematical physics, Springer-Verlag, New York, 1966. MR 38 \#1291.

10. E. Nelson, Analytic vectors, Ann. of Math. (2) 70 (1959), 572-615. MR 21 \#5901. 
11. E. Nelson and W. F. Stinespring, Representation of elliptic operators in an enveloping algebra, Amer. J. Math. 81 (1959), 547-560. MR 22 \#907.

12. R. E. A. C. Paley and N. Wiener, Fourier transforms in the complex domain, Amer. Math. Soc. Colloq. Publ., vol. 19, Amer. Math. Soc., Providence, R. I., 1934; reprint, 1967.

13. N. K. S. Poulsen, Regularity aspects of the theory of infinite dimensional representations of Lie groups, Ph.D. Thesis, M.I.T., Cambridge, Mass., 1970.

14. L. Pukánsky, Leçons sur les représentations des groupes, Monographies de la Société Mathématique de France, no. 2, Dunod, Paris, 1967. MR 36 \#311.

15. W. F. Stinespring, Integrability of Fourier transforms for unimodular Lie groups, Duke Math. J. 26 (1959), 123-131. MR 21 \#2921.

16. K. Yosida, Functional analysis, Die Grundlehren der math. Wissenschaften, Band 123, Academic Press, New York; Springer-Verlag, Berlin, 1965. MR 31 \#5054.

Massachusetts Institute of Technology,

Cambridge, Massachusetts 02139 